\title{
Eudragit as the Material for the Production of Rifampicin and Isoniazid Microparticles
}

\author{
Ng Chai Hwai and Amirah Mohd Gazzali* \\ School of Pharmaceutical Sciences, Universiti Sains Malaysia, \\ 11800 USM Pulau Pinang, Malaysia \\ *Corresponding author: amirahmg@usm.my
}

Published online: 25 December 2019

To cite this article: Ng, C. H. \& Gazzali, A. M. (2019). Eudragit as the material for the production of rifampicin and isoniazid microparticles. J. Phys. Sci., 30(Supp. 2), 221-233, https://doi.org/10.21315/jps2019.30.s2.19

To link to this article: https://doi.org/10.21315/jps2019.30.s2.19

ABSTRACT: Rifampicin and isoniazid are two of the first-line drugs used to treat tuberculosis (TB). Rifampicin is sensitive to light, heat and moisture, and is also known to have poor solubility which affects the absorption of the drug in the gastrointestinal tract. This study was conducted to investigate the effects of different types of Eudragit ${ }^{\circledR}$ in the formulation of microparticles of rifampicin and isoniazid. Two types of Eudragit were used which are Eudragit RL-PO and Eudragit $R S-P O$. The particle size of rifampicin microparticles for Eudragit $R L-P O$ and Eudragit $R S-P O$ were recorded at $44 \mu \mathrm{m}$ and $64 \mu \mathrm{m}$, respectively. Isoniazid microparticles were found to have volume mean diameters of $63 \mu \mathrm{m}$ and $54 \mu \mathrm{m}$ for formulations incorporated with Eudragit RL-PO and Eudragit $R S-P O$, respectively. Eudragit $R L-P O$ formulated rifampicin microparticles encapsulated $80.8 \%$ and $23.3 \%$ for those formulated with Eudragit RS-PO. Isoniazid microparticles recorded an encapsulation efficiency of $98.7 \%$ and $61.9 \%$ in formulations using Eudragit $R L-P O$ and Eudragit RS-PO correspondingly. The formulations retained some of the functional groups, but changes were also observed. Scanning electron microscope (SEM) shows consistently spherical and porous microparticles with rough surfaces. Dissolution studies indicate that isoniazid was released by Quasi-Fickian mechanism while rifampicin was released by non-Fickian mechanism.

Keywords: Eudragit, microparticles, rifampicin, isoniazid, tuberculosis 


\section{INTRODUCTION}

Eudragit $\AA$ is a group of pre-formulated polymers, which are non-toxic, nonabsorbable and non-biodegradable. They are being widely used in the formulation of pharmaceuticals, ranging from the production of conventional granules for tablets to the formulation of advanced delivery products such as sustained-release particles. In the formulation of sustained-release products, Eudragit RL-PO and RS-PO are among the choices available due to their low water solubility and good permeability.

The two aforementioned Eudragit are the copolymer of ethyl acrylate, methyl methacrylate and methacrylic acid ester, with the presence of quaternary ammonium group. The quaternary ammonium group contributes to the water permeability of the Eudragit. The amount of the quaternary ammonium group differs between the two Eudragit, with RL-PO containing more than the RS-PO. This makes RL-PO more water permeable than RS-PO. ${ }^{1}$ RL-PO and RS-PO can be used in the preparation of granules, tablets and other particles. There are reports in the literature on the application of both Eudragit as a material for microparticles and nanoparticles production through different methods such as microencapsulation..$^{2-5}$

Tuberculosis (TB) is an infectious disease caused by bacteria from the Mycobacterium $s p$., and is among the top 10 causes of death worldwide. World Health Organization (WHO) reported that 10 million cases of TB were recorded in 2017, with 1.6 million deaths. ${ }^{6}$ Rifampicin and isoniazid are two of the first line pharmacotherapy choices for TB. ${ }^{5}$ They have been effective in treating TB since the 1950s, although the problem of multidrug resistant TB (MDR-TB) has significantly reduced their effectiveness. The incidence of MDR-TB has been increasing with more cases of rifampicin resistance being detected. Rifampicinresistant strain was detected in $82 \%$ cases of all the MDR-TB. ${ }^{6}$ Among the reasons that may have caused the development of MDR-TB strains is the incomplete treatment by patients due to the long and arduous treatment regimen that they must follow, besides the high number of pills involved throughout the minimum sixmonth treatment period. ${ }^{?}$

Different approaches have been taken to overcome this problem and this can be seen with the development of fixed-dose combination (FDC) involving the anti-TB drugs, which could significantly reduce the number of pills that need to be taken. The FDC which is currently available however, still necessitates the intake of pills every day by patients. With the aim of increasing the treatment effectiveness, the current project has proposed the utilisation of Eudragit RL-PO and RS-PO as the material for the microencapsulation of rifampicin and isoniazid to prolong the drug 
release of both drugs, which could lead to the reduction of the dosing regimen and the amount of drug needed for each dose. This will benefit patients by reducing the risk of side effects, improving their compliance to the medication and ultimately reduce the cases of MDR-TB.

\section{EXPERIMENTAL}

\subsection{Materials}

Rifampicin was obtained from Bio Basic Inc. (Canada), while isoniazid and polyvinyl alcohol (PVA) were purchased from BDH Laboratory Reagents, Poole (United Kingdom). Eudragit RL-PO and Eudragit RS-PO were acquired from Röhm GmbH, Germany. Dichloromethane (AR grade) was obtained from QRëC (Quality Reagent Chemical) Asia. The chemicals were used as received without further purification.

\subsection{Methods}

\subsubsection{Production of isoniazid and rifampicin microparticles}

The microparticles were produced as described in the literature with certain modifications. ${ }^{2}$ Briefly, $1 \mathrm{~g}$ of PVA was solubilised in $100 \mathrm{ml}$ distilled water. Separately, $0.8 \mathrm{~g}$ of Eudragit (RL-PO or RS-PO) was added with $0.2 \mathrm{~g}$ of drug (isoniazid or rifampicin) before being homogenously mixed with $8 \mathrm{ml}$ of dichloromethane. The PVA solution was added into the Eudragit-drug mixture and stirred for $5 \mathrm{~h}$ to remove the solvent. The resultant microparticles were obtained through lyophilisation for $48 \mathrm{~h}$ followed by sieving through a $250 \mu \mathrm{m}$ sieve.

\subsubsection{Physicochemical characterisation of the microparticles}

The physicochemical characterisation of the microparticles was conducted in terms of particle size, percentage of drug encapsulation, percentage of yield, surface morphology, infrared analysis and in-vitro drug release study.

\subsubsection{Particle size distribution}

The particle size distribution (PSD) of each sample was analysed using Malvern Instruments S Longbed-MAM5005 Master Sizer Particle Size Analyzer (version 2.19, United Kingdom). Approximately $5 \mathrm{ml}$ of well-dispersed sample was introduced into the dispersion unit with water as the dispersant. The speed of pump 
stirrer was set at $1800 \mathrm{rpm}$. The laser diffraction system equipped with appropriate lens for the sample's particle-size range was used to measure the PSD of the sample. Each sample was tested in triplicate.

\subsubsection{Percentage of encapsulation efficiency}

The encapsulation efficiency (\%) of the microparticles was calculated using Equation 1 as described in the literature..$^{8,9}$

$$
E E(\%)=\frac{W_{A}}{W_{D}} \times 100
$$

where $W_{A}$ is the actual amount of drug encapsulated while $W_{D}$ is the amount of drug incorporated in the formulation.

\subsubsection{Preparation of calibration curves}

The calibration curves for isoniazid and rifampicin were prepared in water and acetone, respectively. A stock solution containing $1 \mathrm{mg} \mathrm{ml}^{-1}$ of each drug was prepared and a serial dilution was made accordingly. The absorbance of each concentration was measured by using a UV-visible spectrophotometer (HITACHI U-2000 Spectrophotometer) at $266 \mathrm{~nm}$ (isoniazid) and $475 \mathrm{~nm}$ (rifampicin) in triplicates. A calibration curve was then plotted for each drug.

\subsubsection{Scanning electron microscopy}

The surface morphology of the microparticles was visualised using ZEISS SUPRA ${ }^{\circledR} 50$ VP scanning electron microscope (SEM). The samples were precoated with a layer of gold $(\mathrm{Au})$ as a standard to improve image resolution and contrast in the field transmission system to produce a homogenous surface for imaging. 10

\subsubsection{Fourier transform infrared spectroscopy (FTIR)}

The potassium bromide $(\mathrm{KBr})$ technique was used in the sample preparation of pure drugs, excipients and the microparticles. Approximately $1 \mathrm{mg}$ of test sample was mixed with $99 \mathrm{mg}$ of $\mathrm{KBr}$ by triturating and grinding in a mortar with the use of a pestle. The powder mixture was then compressed with a compaction force of 5 tons followed by 10-15 s of holding by using a hydraulic press pump (BIORAD, United States). Each sample was scanned 32 times and the spectra produced with percentage transmission versus wavenumbers were then analysed by using Omnic software (Thermo Nicolet, United States). 


\subsubsection{In-vitro drug release study}

The in-vitro drug release from the formulated microparticles was studied in a phosphate-buffered solution ( $\mathrm{pH}$ 6.8). USP Apparatus 1 with rotating baskets was set to sink conditions during the study. An amount of $50 \mathrm{mg}$ of each microparticles was placed in the dissolution medium maintained at $37^{\circ} \mathrm{C} \pm 0.5^{\circ} \mathrm{C}$. At each time interval, $10 \mathrm{ml}$ of sample was withdrawn and an equivalent of $10 \mathrm{ml}$ fresh dissolution medium was replaced to maintain the sink condition. The amount of drug released was determined spectrophotometrically (HITACHI U-2000 spectrophotometer) at $266 \mathrm{~nm}$ for isoniazid and $475 \mathrm{~nm}$ for rifampicin based on the standard curve prepared earlier. The experiment was done in triplicates.

\section{RESULTS AND DISCUSSION}

\subsection{Production and Physicochemical Characterisation of Microparticles}

The particle size distribution was evaluated through determining the volume moment mean, or also known as the De Brouckere Mean Diameter, of the microparticles produced. This parameter was chosen because it reflects the size of the particles that constituted the bulk of the sample volume. By using the weighted volume moment mean diameter, it is assumed that the spherical particles are of the same volume as the actual particles. ${ }^{11}$ Figure 1 shows the volume moment mean diameters of microparticles obtained from the analysis.

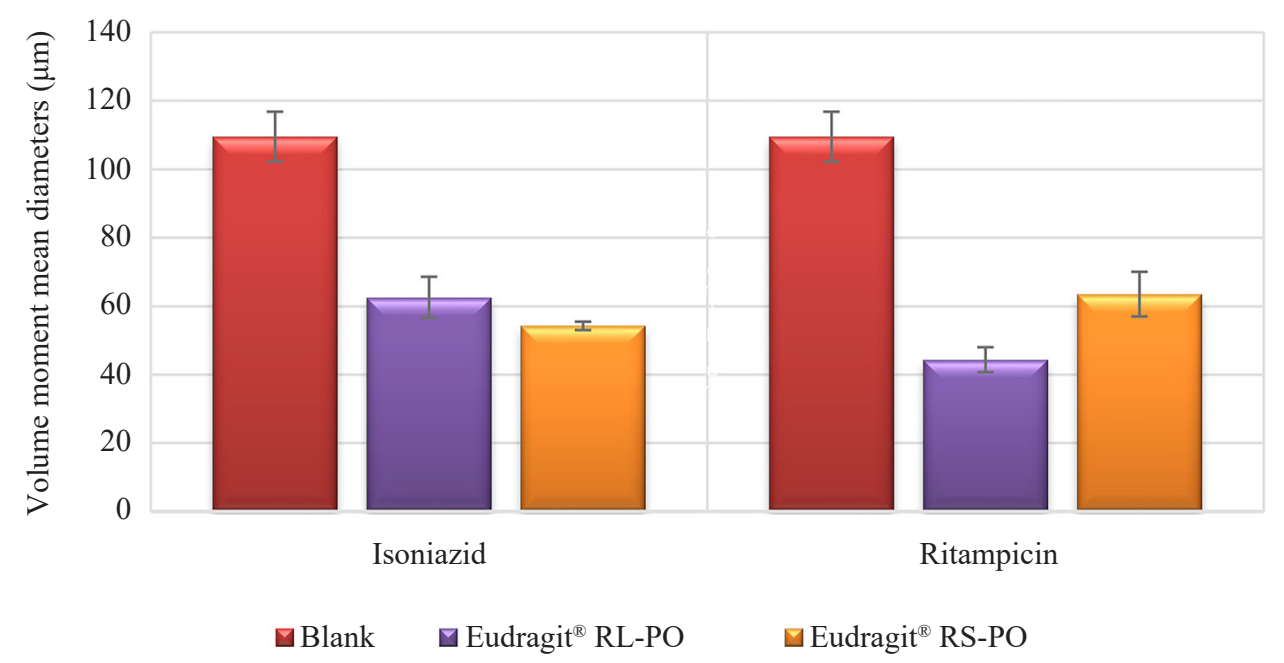

Figure 1: Volume moment mean diameter of microparticles produced $(\mathrm{n} \geq 2)$. 
Based on Figure 1, on average, the microparticles loaded with drug were found to have lower particle size, between $44 \mu \mathrm{m}$ and $64 \mu \mathrm{m}$, whereas the blank microparticles were found to have bigger particle size recorded at $110 \mu \mathrm{m}$. It was hypothesised that this phenomenon is due to the compaction of the particles during the production step, which may be induced by the incorporated drugs. The drugs may improve the network of the polymers, which will produce smaller particles as a result. ${ }^{12}$ The blank microparticles of Eudragit RL-PO and RS-PO has shown similar particle size, in accordance to the finding reported by Nath et al. ${ }^{13}$

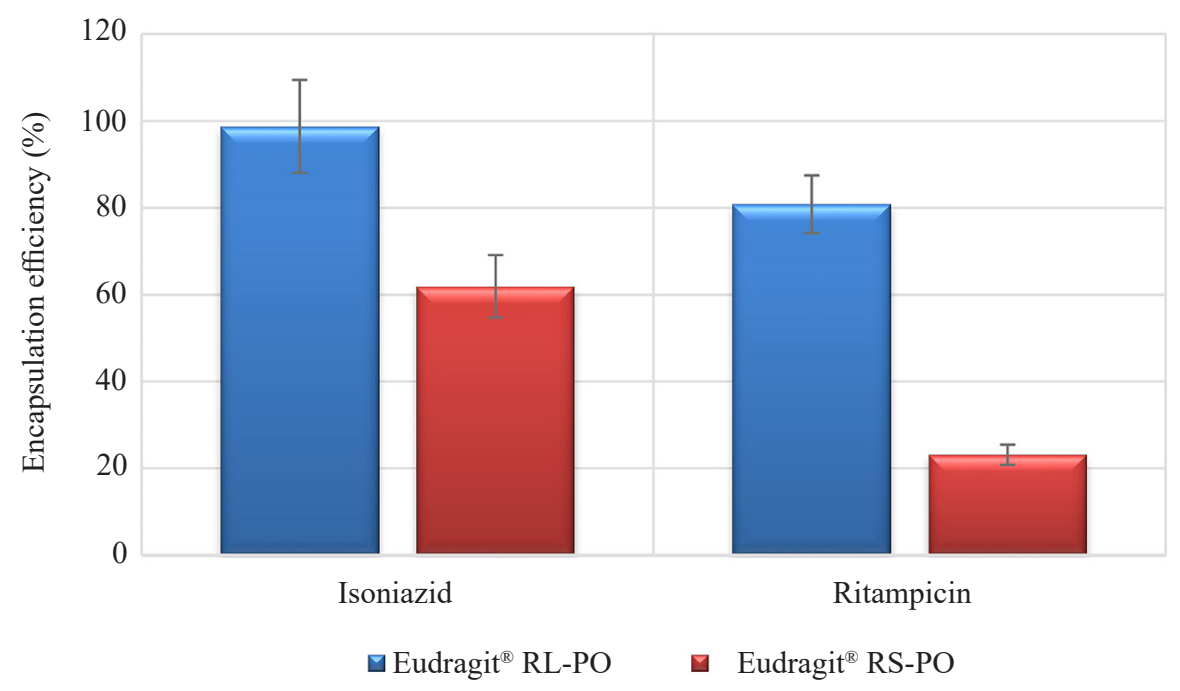

Figure 2: Encapsulation efficiency (EE\%) of isoniazid and rifampicin microparticles.

The microparticles produced from Eudragit RL-PO show a significantly higher encapsulation percentage as compared to Eudragit RS-PO for both drugs $(p<0.05)$ (Figure 2). This means that for rifampicin, the encapsulation by RL-PO was much favourable than RS-PO, which is the same for isoniazid. Due to the fact that the difference between the two polymers are only the amount of quaternary ammonium group, it could be hypothesised that the presence of this group has influenced the encapsulation of drugs in the microparticles. When comparing the same polymer encapsulation percentage, the difference between the amount of both drugs encapsulated by RL-PO is not statistically significant $(p>0.05)$, which shows that the encapsulation ability of RL-PO for both hydrophilic (isoniazid) and hydrophobic (rifampicin) drugs might be similar. On the other hand, the EE\% of RS-PO is statistically significant between the two drugs $(p<0.05)$, indicating that for RS-PO, more isoniazid is present in the microparticles, perhaps through deposition on the surface of the particles. 
The surface morphology of the microparticles as captured by the SEM is presented in Figure 3. As seen in the figure, the microparticles produced are in a uniformly round shape with high porosity.
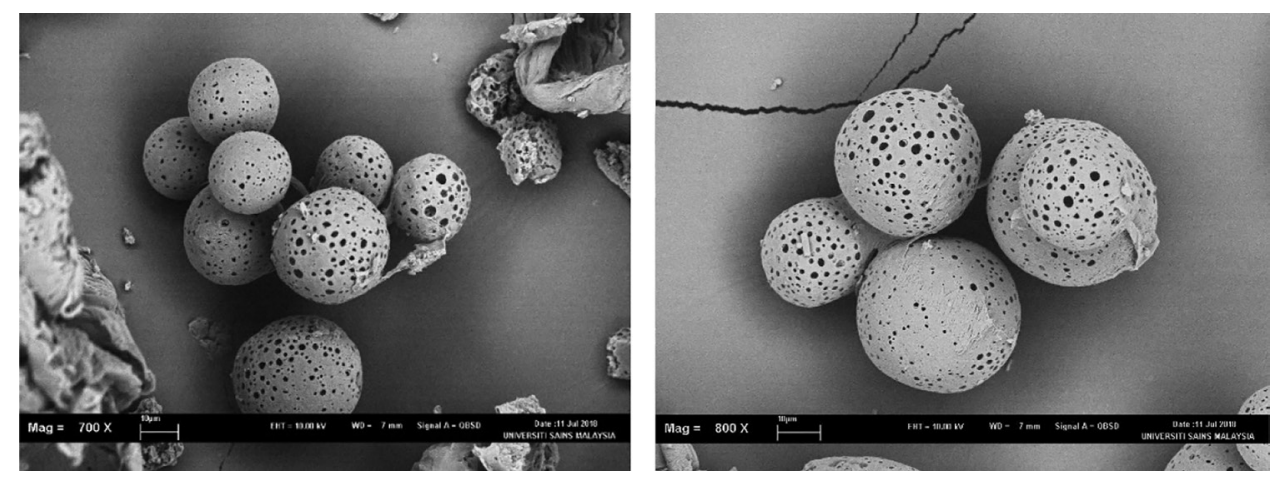

Figure 3: SEM images of the microparticles produced.

The microparticles appeared to be porous and possess rough surfaces. The pores on the microparticles were observed to be irregularly sized. These pores were formed during the evaporation of solvents from the formulation through the surface of the microparticles. In addition, some of the microparticles were found to have bound with tiny fragments of particulates, which are postulated to be free drug particles.

In general, the composition of Eudragit polymer present in microparticles plays a significant role in determining the degree of porosity of microparticles, in which a high content of Eudragit polymer will lead to the loss of porosity. Based on data available in the literature, drug to polymer ratio ranging from 1:1 to 1:4 would usually yield stable and porous microparticles. ${ }^{14}$ The ratio 1:4 was used in the preparation of microparticles in this study.

\subsubsection{FTIR}

Following the production of rifampicin- and isoniazid-loaded microparticles, several significant shifts were identified from the FTIR spectra of the microparticles.

The FTIR data of rifampicin-loaded Eudragit RL-PO and RS-PO is summarised in Tables 1 and 2, respectively. The absorption band of $\mathrm{O}-\mathrm{H}$ and $\mathrm{N}-\mathrm{H}$ at $3449 \mathrm{~cm}^{-1}$ was shifted to $3388 \mathrm{~cm}^{-1}$ and $3406 \mathrm{~cm}^{-1}$ in RS-PO and RL-PO formulations. Apart from that, the absorption intensities were higher compared to pure rifampicin perhaps due to the presence of PVA and the Eudragit. 
Table 1: The FTIR absorbance bands of polymer (Eudragit RL-PO), blank and rifampicinloaded microparticles.

\begin{tabular}{lcccc}
\hline & \multicolumn{4}{c}{ Absorption bands $\left(\mathrm{cm}^{-1}\right)$} \\
\cline { 2 - 5 } & Rifampicin & $\begin{array}{c}\text { Eudragit } \\
\text { RL-PO }\end{array}$ & Blank & $\begin{array}{c}\text { Rifampicin-loaded } \\
\text { microparticles }\end{array}$ \\
\cline { 2 - 5 } $\begin{array}{l}\text {-OH and -NH } \\
\text { stretching }\end{array}$ & 3449 & 3444.7 & 3443.3 & 3388.0 \\
$-\mathrm{CH}$ stretching & $\begin{array}{r}2970.5 \mathrm{~cm}^{-1}, 2936.6 \mathrm{~cm}^{-1} \\
\text { and } 2881.6\end{array}$ & 2954.4 & 2950.3 & 2946.2 \\
C=O acetyl & 1732.2 and 1712.0 & 1737.5 & 1735.2 & 1736.9 \\
stretching & 1655.2 & 1634.7 & & \\
$\mathrm{C}=\mathrm{N}$ asymmetric & 1567.0 & & & \\
$\mathrm{C}=\mathrm{C}$ stretching & 1374.6 & 1386.9 & 1383.4 & 1378.6 \\
$\mathrm{C}-\mathrm{N}$ bending & 1252.3 & 1246.2 & 1257.5 & 1245.4 \\
$\mathrm{C}-\mathrm{O}-\mathrm{C}$ bending & & 1147.2 & 1144.3 & 1145.7 \\
$\mathrm{C}-\mathrm{O}-\mathrm{C}$ stretching & 973.4 & 987.2 & & \\
$\mathrm{C}=\mathrm{C}$ bending & & & & \\
\hline
\end{tabular}

Table 2: The FTIR absorbance bands of polymer (Eudragit RS-PO), blank and rifampicinloaded microparticles.

\begin{tabular}{|c|c|c|c|c|}
\hline & \multicolumn{4}{|c|}{ Absorption bands $\left(\mathrm{cm}^{-1}\right)$} \\
\hline & Rifampicin & $\begin{array}{l}\text { Eudragit } \\
\text { RS-PO }\end{array}$ & Blank & $\begin{array}{l}\text { Rifampicin-loaded } \\
\text { microparticles }\end{array}$ \\
\hline $\begin{array}{l}-\mathrm{OH} \text { and }-\mathrm{NH} \\
\text { stretching }\end{array}$ & 3449 & 3456.1 & 3443.3 & 3406.0 \\
\hline -CH stretching & $\begin{array}{c}2970.5 \mathrm{~cm}^{-1}, 2936.6 \mathrm{~cm}^{-1} \\
\text { and } 2881.6\end{array}$ & 2993.9 & 2950.3 & 2948.6 \\
\hline $\begin{array}{l}\mathrm{C}=\mathrm{O} \text { acetyl } \\
\text { stretching }\end{array}$ & $1732.2 \mathrm{~cm}^{-1}$ and 1712.0 & 1736.5 & 1735.2 & 1737.4 \\
\hline $\mathrm{C}=\mathrm{N}$ asymmetric & 1655.2 & & & \\
\hline $\mathrm{C}=\mathrm{C}$ stretching & 1567.0 & & & \\
\hline $\mathrm{C}-\mathrm{N}$ bending & 1374.6 & 1386.9 & 1383.4 & 1380.9 \\
\hline $\mathrm{C}-\mathrm{O}-\mathrm{C}$ bending & 1252.3 & 1246.2 & 1257.5 & 1244.6 \\
\hline C-O-C stretching & & 1148.6 & 1144.3 & 1145.5 \\
\hline $\mathrm{C}=\mathrm{C}$ bending & 973.4 & 993.5 & & \\
\hline
\end{tabular}


The FTIR spectrum of isoniazid-based microparticles from Eudragit ${ }^{\circledR}$ RLPO (Table 3) and RS-PO (Table 4) showed that the -OH and -NH stretching remained in both formulations. $\mathrm{CH}$ and $\mathrm{C}-\mathrm{O}$ acetyl stretching were observed in the formulations, which did not appear in the spectrum of pure isoniazid. The C-N bending, $\mathrm{C}-\mathrm{O}-\mathrm{C}$ bending, $\mathrm{C}-\mathrm{O}-\mathrm{C}$ stretching and $\mathrm{C}=\mathrm{C}$ bending remained in both isoniazid formulations.

Table 3: The FTIR absorbance bands of polymer (Eudragit RL-PO), blank and isoniazidloaded microparticles.

\begin{tabular}{lcccc}
\hline & \multicolumn{4}{c}{ Absorption bands $\left(\mathrm{cm}^{-1}\right)$} \\
\cline { 2 - 5 } & Isoniazid & $\begin{array}{c}\text { Eudragit } \\
\text { RL-PO }\end{array}$ & Blank & $\begin{array}{c}\text { Isoniazid-loaded } \\
\text { microparticles }\end{array}$ \\
\hline -OH and -NH stretching & 3303.7 & 3444.7 & 3443.3 & 3389.7 \\
-CH stretching & & 2954.4 & 2950.3 & 2947.5 \\
C=O acetyl stretching & 1667.0 & 1634.7 & 1735.2 & 1737.4 \\
C=N asymmetric & 1555.4 & & & 1666.8 \\
C=C stretching & 1334.3 & 1386.9 & 1383.4 & 1562.8 \\
C-N bending & 1221.3 & 1246.2 & 1257.5 & 1379.6 \\
C-O-C bending & 1142.0 & 1147.2 & 1144.3 & 1144.1 \\
C-O-C stretching & 995.4 & 987.2 & & 995.5 \\
C=C bending & & & & \\
\hline
\end{tabular}

Table 4: The FTIR absorbance bands of polymer (Eudragit RS-PO), blank and isoniazidloaded microparticles.

\begin{tabular}{lcccc}
\hline & \multicolumn{4}{c}{ Absorption bands $\left(\mathrm{cm}^{-1}\right)$} \\
\cline { 2 - 5 } & Isoniazid & $\begin{array}{c}\text { Eudragit } \\
\text { RS-PO }\end{array}$ & Blank & $\begin{array}{c}\text { Isoniazid-loaded } \\
\text { microparticles }\end{array}$ \\
\hline -OH and -NH stretching & 3303.7 & & 3443.3 & 3433.4 \\
-CH stretching & & 2993.9 & 2950.3 & 2991.7 \\
C=O acetyl stretching & 1667.0 & 1736.5 & 1735.2 & 1733.3 \\
C=N asymmetric & 1555.4 & & & \\
C=C stretching & 1334.3 & 1386.9 & 1383.4 & 1385.3 \\
C-N bending & 1221.3 & & 1257.5 & 1243.9 \\
C-O-C bending & 1142.0 & 1148.6 & 1144.3 & 1146.1 \\
C-O-C stretching & 995.4 & 993.5 & & 994.4 \\
C=C bending & & & & \\
\hline
\end{tabular}




\subsection{In-vitro Drug Release Study}

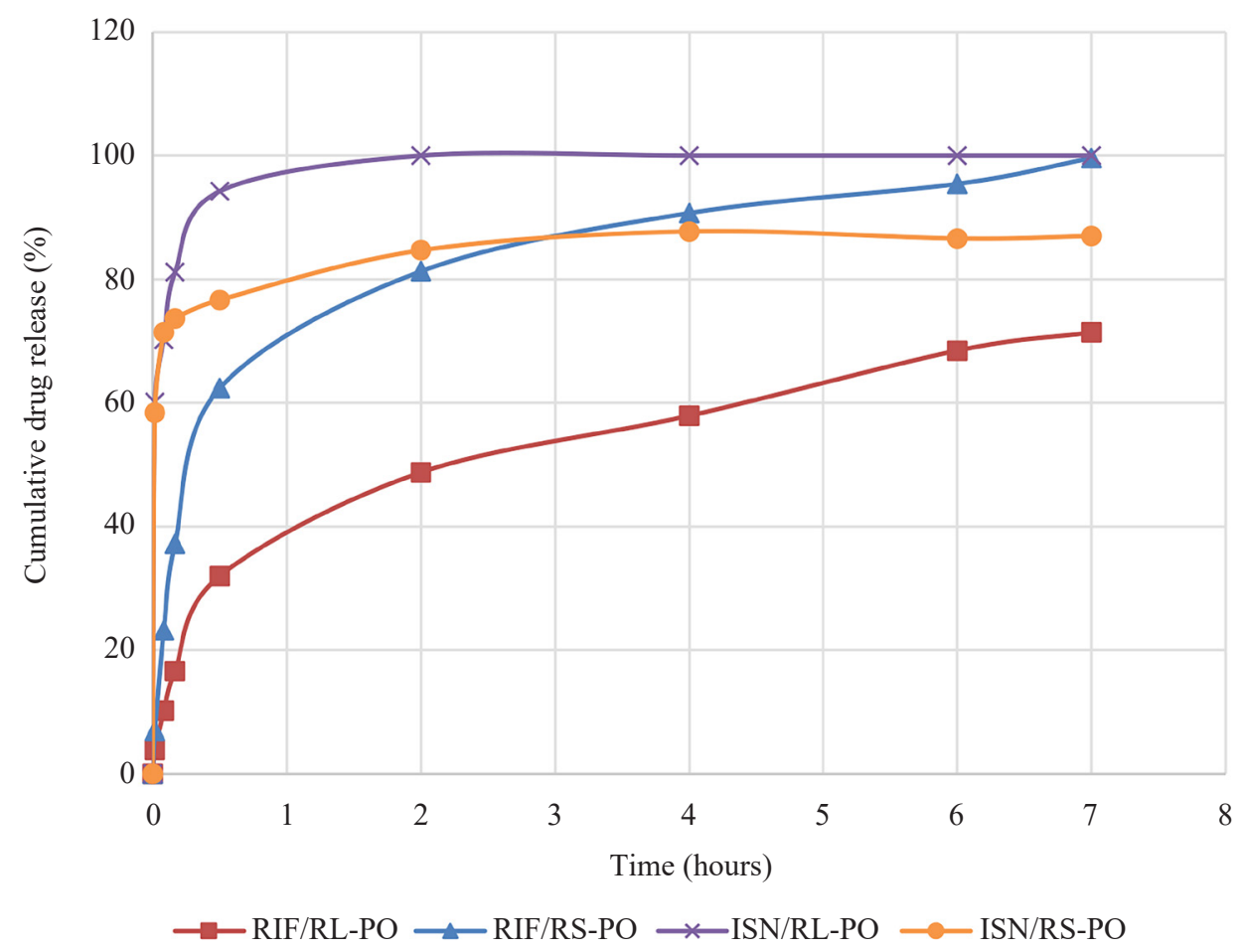

Figure 4: Release of rifampicin and isoniazid from Eudragit RL-PO and RS-PO.

Figure 4 shows the release characteristics of the encapsulated rifampicin and isoniazid from both polymers. The release of isoniazid from both polymers is characterised by a burst release followed by a more stable release until the end of the study.

Isoniazid encapsulated in Eudragit RL-PO showed a faster release compared to RS-PO, which is due to the high water permeability of the RL-PO polymer. ${ }^{15}$ The higher burst release observed for isoniazid could also be due to the presence of the drug molecules on the surface of the microparticles. This could be correlated to the high encapsulation efficiency of isoniazid in both polymers, in which the drug might be deposited on the outer surface of the microparticles instead of being encapsulated within. This is expected for isoniazid as the drug is hydrophilic in nature, hence encapsulation within the particles is more challenging as compared to hydrophobic drugs. ${ }^{16}$ In addition, the fact that RL-PO grade swells more than the RS-PO grade may also contribute to the faster release observed in the case of isoniazid. ${ }^{17}$ 
Compared to isoniazid, the rate of release of rifampicin from RL-PO is comparatively slower than the RS-PO. This might be due to the more hydrophilic nature of RL-PO compared to RS-PO, which renders inherent water permeability, allowing a more water-soluble drug like isoniazid to diffuse better through the hydrated polymeric network. ${ }^{18}$

Many factors may influence the rate of drug release from microparticles and one of the factors is the dissolution of polymer coat in the environment liquid, which in this case is the buffer ( $\mathrm{pH}$ 6.8). However, since both Eudragit RL-PO and RS-PO exhibit $\mathrm{pH}$ independent, time-controlled release properties, $\mathrm{pH}$ is not a considerable factor that can affect the release of the encapsulated drug. ${ }^{19}$

The data obtained from the in-vitro drug release study were fitted in different kinetics model to further explain the release characteristics of the encapsulated drug from the microparticles. Table 5 shows the $\mathrm{R}^{2}$ value obtained.

Table 5: The $\mathrm{R}^{2}$ values obtained from fitting the in-vitro drug release data into different release kinetic models.

\begin{tabular}{lccccc}
\hline & Zero-order & First-order & Hixson-Crowell & Higuchi's & Korsmeyer-Peppas \\
\hline ISN/RL-PO & 0.5057 & 0.8734 & 0.9953 & 0.9887 & $0.9578(n=0.12)$ \\
ISN/RS-PO & 0.6334 & 0.7358 & 0.7923 & 0.9117 & $0.9576(n=0.11)$ \\
RIF/RL-PO & 0.8726 & 0.9518 & 0.9650 & 0.9922 & $0.9984(n=0.63)$ \\
RIF/RS-PO & 0.7359 & 0.9182 & 0.9402 & 0.9985 & $0.9995(n=0.73)$ \\
\hline
\end{tabular}

The $\mathrm{R}^{2}$ values of first-order kinetics were higher than zero-order kinetics for all formulations, showing that the release of both rifampicin and isoniazid from the microparticles is dependent on its concentration. The Higuchi's equation shows high linearity for both rifampicin-encapsulated microparticles, indicating that the release was governed by diffusion more than dissolution. The $n$ values calculated for isoniazid from both polymers in the Korsmeyer-Peppas models shows that the release occured through Quasi-Fickian diffusion $(n<0.5)$, which means that the matrix did not swell (non-swellable matrix diffusion). On the other hand, the $n$ values calculated for the release of rifampicin were higher than 0.5 , indicating that the drug was transported through non-Fickian mechanism, which is through diffusion and erosion of the polymer matrix. This is in accordance with the slower release rate of rifampicin as compared with isoniazid, as discussed earlier. The non-Fickian mechanism is a common method for the release of hydrophobic drug such as rifampicin, as reported by other publications. ${ }^{20,21}$ 
From the kinetic analysis, it could be deduced that the release of encapsulated isoniazid did not necessitate the swelling of the matrix, most probably due to the deposition of the drug on the particle surface. The release of rifampicin on the other hand need the swelling and erosion of the matrix, probably because it was embedded in the matrix during microparticles production.

\section{CONCLUSION}

Eudragit RL-PO and RS-PO are potentially effective polymers in modifying the release of hydrophobic drugs such as rifampicin and less for hydrophilic drugs such as isoniazid. Further studies are required and the combination of both polymers in one formula could be an option to enhance their function, perhaps as sustained release delivery model. With more research-based evidence, Eudragit RL-PO and RS-PO might be a good option to produce efficient drug delivery system to enhance TB treatment in the future.

\section{ACKNOWLEDGEMENTS}

The authors would like to acknowledge the contribution by Mr. Goh Gee Seng in this research work.

\section{REFERENCES}

1. Apu, S. A. et al. (2009). Investigation of in vitro release kinetics of carbamazepine from Eudragit ${ }^{\circ}$ RS PO and RL PO matrix tablets. Trop. J. Pharm. Res., 8(2), 145-152, https://doi.org/10.4314/tjpr.v8i2.44523.

2. Cortesi, R. et al. (2012). Eudragit( (®) microparticles for the release of budesonide: A comparative study. Ind. J. Pharm. Sci., 74(5), 415-421.

3. Pradhan, R. et al. (2016). Preparation and characterization of spray-dried valsartanloaded Eudragit ${ }^{\circledR}$ E PO solid dispersion microparticles. Asian J. Pharm. Sci., 11(6), 744-750, https://doi.org/10.1016/j.ajps.2016.05.002.

4. Soltani, S. et al. (2016). Design of eudragit RL nanoparticles by nanoemulsion method as carriers for ophthalmic drug delivery of ketotifen fumarate. Iran $J$. Basic Med. Sci., 19(5), 550-560.

5. Tang, J. et al. (2011). Eudragit nanoparticles containing genistein: Formulation, development, and bioavailability assessment. Int. J. Nanomed., 6, 2429-2435.

6. World Health Organization (WHO). (2017). Towards ending tuberculosis: What gets measured gets done. Geneva: WHO.

7. Aziah, A. M. (2004). Tuberculosis in Malaysia: Combating the old nemesis. Med. J. Mal., 59(1), 1-3. 
8. Dey, S., Pramanik, S. \& Malgope, A. (2011). Formulation and optimization of sustained release stavudine microspheres using response surface methodology. ISRN Pharm., 11, Article ID 627623, https://doi.org/10.5402/2011/627623.

9. El-Say, K. M. (2016). Maximizing the encapsulation efficiency and the bioavailability of controlled-release cetirizine microspheres using Draper-Lin small composite design. Drug Des. Dev. Ther., 10, 825-839, https://doi.org/10.2147/ dddt.s101900.

10. Leslie, S. A. \& Mitchell, J. C. (2007). Removing gold coating from SEM samples. Palaeontol., 50(6), 1459-1461, https://doi.org/10.1111/j.1475-4983.2007.00718.x.

11. Mingard, K. et al. (2009). Measurement good practice guide no. 111. Middlesex: National Physical Laboratory.

12. Bansode, S. S. et al. (2010). Microencapsulation: A review. Int. J. Pharm. Sci. Rev. Res., 1(2), 38-43.

13. Nath, B., Nath. L. K. \& Kumar, P. (2011). Preparation and in vitro dissolution profile of zidovudine loaded microspheres made of Eudragit RS 100, RL 100 and their combinations. Acta Polon. Pharm., 68(3), 409-415.

14. Bhise, S. B., More, A. B. \& Malayandi, R. (2010). Formulation and in vitro evaluation of rifampicin loaded porous microspheres. Scient. Pharm., 78(2), 291-302, https://doi.org/10.3797/scipharm.0910-09.

15. Boyapally, H., Nukala, R. K. \& Douroumis, D. (2009). Development and release mechanism of diltiazem $\mathrm{HCl}$ prolonged release matrix tablets. Drug Del., 16(2), 67-74, https://doi.org/10.1080/10717540802586220.

16. Seyfoddin, A, Shaw, J. \& Al-Kassas, R. (2010). Solid lipid nanoparticles for ocular drug delivery. Drug Del., 17, 467-489, https://doi.org/10.3109/10717544.2010.4 83257.

17. Das, S. K. \& Das, N. G. (1998). Preparation and in vitro dissolution profile of dual polymer (Eudragit RS100 and RL100) microparticles of diltiazem hydrochloride. J. Microencap., 15(4), 445-452, https://doi.org/10.3109/02652049809006871.

18. Quinten, T. et al. (2012). Preparation and evaluation of sustained-release matrix tablets based on metoprolol and an acrylic carrier using injection moulding. AAPS PharmSciTech, 13(4), 1197-1211, https://doi.org/10.1208/s12249-012-9848-6.

19. Evonik Industries. (2010). Eudragit $\AA$ setting benchmarks in oral solid dosage forms since 1954. Darmstadt: Evonik Industries.

20. Mei, Q. et al. (2018). Formulation and in vitro characterization of rifampicinloaded porous poly ( $\varepsilon$-caprolactone) microspheres for sustained skeletal delivery. Drug Desd Dev. Ther., 12, 1533-1544, https://doi.org/10.2147/dddt.s163005.

21. Paramita, C. \& Uma Shankar, M. S. (2012). Formulation and evaluation of rifampicin and ofloxacin niosomes for drug-resistant TB on logarithmic-phase cultures of Mycobacterium tuberculosis. Int. J. Res. Pharm. Sci., 3(4), 628-633. 